Ralf Dreyer

Die Arabische Republik Jemen

Zur Verfassung und Verwaltung eines Entwicklungslandes

Studienverlag N. Brockmeyer, Bochum, 1983, XI und 212 S., DM 29,80

Die vorliegende Arbeit gliedert sich in eine Einführung in die ökonomische und soziale Struktur des Jemen, die Beschreibung der Verfassungs- und Verwaltungsstruktur zur Zeit des Imamats (bis zum Bürgerkrieg von 1962-1969) und die Darstellung der Verfassungsentwicklung von 1962-1976. Dreyer hat über die politisch-administrative und konstitutionelle Entwicklung des Jemen, der bekanntlich bis zum Bürgerkrieg nach außen hin fast hermetisch abgeschlossen war, umfangreiches Material zusammengetragen. Die Fülle der Einzelheiten, insbesondere hinsichtlich der konstitutionellen Entwicklung, macht sein Buch hilfreich für jeden, der sich über diesen Aspekt des modernen Nordjemen informieren will. Schon der Aufbau der Arbeit, der sich stark an der historischen Entwicklung orientiert, macht deutlich, daß Darstellung, nicht Analyse, das Hauptanliegen der Arbeit ist. Dieser erste Eindruck nach einem Blick in das Inhaltsverzeichnis erweist sich als richtig, obwohl der Autor selbst in der Einleitung recht hohe theoretische Ansprüche anmeldet. Der Leser sollte sich hiervon nicht täuschen lassen, denn Dreyer kann diesen Ansprüchen nicht gerecht werden: Die Basis sozialwissenschaftlicher Literatur, auf der seine Arbeit aufbaut, ist viel zu schmal, als daß er sozialwissenschaftliche Ansätze wirklich für den Fall Jemen fruchtbar machen könnte (symptomatisch die Berufung auf Zippelius, Allgemeine Staatslehre als einziger Literatur zur politischen Systemtheorie, deren Ansatz Dreyer übernehmen und operationalisieren will (Einleitung S. XI) und die unreflektierte Verwendung des Modernisierungsbegriffs). Dementsprechend sind Dreyers Ergebnisse nicht immer plausibel, sie erscheinen bisweilen als bloße Behauptungen. Neben der schmalen theoretischen Literaturbasis enttäuscht auch die Tatsache, daß fast alle im Literaturverzeichnis selbständig genannten arabischen Werke im Text nur indirekt (nach deutschsprachigen Büchern) zitiert werden.

Ulrich Deffaa

\title{
Doris Köhn
}

\section{Die Verschuldung lateinamerikanischer Länder}

Weltarchiv Verlag Hamburg, 1984, 148 S., DM 42,-

In diesem Buch wird ein Ưberblick über die externe Verschuldungslage von ausgewählten Ländern Lateinamerikas während der Zeit von 1973 bis 1982 gegeben. Der erste Teil vermittelt einen Einblick in die theoretischen Grundlagen für die Analyse der Auslandsverschuldung aus makroökonomischer Sicht. Im zweiten Teil wird die Verschuldungssituation der Hauptschuldnerländer Lateinamerikas, Brasilien, Mexiko, Argenti- 